\title{
Fast electron transfer through a single molecule natively structured redox protein
}

Della Pia, Eduardo Antonio ; Chi, Qijin; Macdonald, J. Emyr; Ulstrup, Jens; Jones, D. Dafydd ; Elliott, Martin

\section{Published in:}

Nanoscale

Link to article, DOI:

$10.1039 / \mathrm{c} 2 \mathrm{nr} 32131 \mathrm{a}$

Publication date:

2012

Document Version

Publisher's PDF, also known as Version of record

Link back to DTU Orbit

Citation (APA):

Della Pia, E. A., Chi, Q., Macdonald, J. E., Ulstrup, J., Jones, D. D., \& Elliott, M. (2012). Fast electron transfer through a single molecule natively structured redox protein. Nanoscale, 4(22), 7106-7113.

https://doi.org/10.1039/c2nr32131a

\section{General rights}

Copyright and moral rights for the publications made accessible in the public portal are retained by the authors and/or other copyright owners and it is a condition of accessing publications that users recognise and abide by the legal requirements associated with these rights.

- Users may download and print one copy of any publication from the public portal for the purpose of private study or research.

- You may not further distribute the material or use it for any profit-making activity or commercial gain

- You may freely distribute the URL identifying the publication in the public portal 


\title{
Fast electron transfer through a single molecule natively structured redox protein $\dagger$
}

\author{
Eduardo Antonio Della Pia, $\leftarrow^{a c}$ Qijin Chi, ${ }^{* b}$ J. Emyr Macdonald, ${ }^{a}$ Jens Ulstrup, ${ }^{b}$ D. Dafydd Jones ${ }^{* c}$ \\ and Martin Elliott*a
}

Received 3rd August 2012, Accepted 23rd September 2012

DOI: $10.1039 / \mathrm{c} 2 \mathrm{nr} 32131 \mathrm{a}$

The electron transfer properties of proteins are normally measured as molecularly averaged ensembles. Through these and related measurements, proteins are widely regarded as macroscopically insulating materials. Using scanning tunnelling microscopy (STM), we present new measurements of the conductance through single-molecules of the electron transfer protein cytochrome $b_{562}$ in its native conformation, under pseudo-physiological conditions. This is achieved by thiol (SH) linker pairs at opposite ends of the molecule through protein engineering, resulting in defined covalent contact between a gold surface and a platinum-iridium STM tip. Two different orientations of the linkers were examined: a long-axis configuration (SH-LA) and a short-axis configuration (SH-SA). In each case, the molecular conductance could be 'gated' through electrochemical control of the heme redox state. Reproducible and remarkably high conductance was observed in this relatively complex electron transfer system, with single-molecule conductance values peaking around $18 \mathrm{nS}$ and $12 \mathrm{nS}$ for the SHSA and SH-LA cytochrome $b_{562}$ molecules near zero electrochemical overpotential. This strongly points to the important role of the heme co-factor bound to the natively structured protein. We suggest that the two-step model of protein electron transfer in the STM geometry requires a multi-electron transfer to explain such a high conductance. The model also yields a low value for the reorganisation energy, implying that solvent reorganisation is largely absent.

\section{Introduction}

Long range electron transfer is central to processes essential to life, including photosynthesis, respiration and many enzymatic catalytic events. ${ }^{1,2}$ Electron transfer (ET) proteins are the components responsible for these processes and perform their function through binding, organising and tuning redox active metals and co-factors. To date, most experimental studies on ET through proteins have been achieved by a combination of chemical modification and spectroscopic measurement. ${ }^{3,4}$ These have largely been interpreted in terms of transport through a relatively isotropic dielectric medium, well-approximated by widely applicable parameters, ${ }^{5}$ or a through-bond model in

${ }^{a}$ School of Physics and Astronomy, Cardiff University, Cardiff CF24 3AA, UK.E-mail: martin.elliott@astro.cf.ac.uk; Tel: +44(0)2920875468

${ }^{b}$ Department of Chemistry and NanoDTU, Technical University of Denmark, DK-2800 Lyngby, DK. E-mail: cq@kemi.dtu.dk

'School of Biosciences, Cardiff University, Cardiff CF10 3AT, UK. E-mail: jonesdd@cf.ac.uk

$\dagger$ Electronic supplementary information (ESI) available: Experimental methods, DNA and protein sequences, additional STM statistical analysis and images, electrochemical data and $I_{\mathrm{t}}-z$ data analysis. See DOI: $10.1039 / \mathrm{c} 2 \mathrm{nr} 32131 \mathrm{a}$

t Present address: School of Chemistry, University of Copenhagen, Universitetparken 5, 2100 Copenhagen, DK. which the detailed protein structure influences electron transport to a significant extent. ${ }^{3,4}$

High resolution tools, such as mechanically controlled break junctions, scanning tunnelling microscopy (STM)-based methods and conducting atomic force microscopy (cAFM), have greatly advanced our ability to investigate single molecule conduction and have been widely applied to a range of small organic and inorganic single molecules. ${ }^{6-15}$ However, to apply these tools to measure reliably electronic conductance of structurally complex, and mechanically fragile, single protein molecules, several conditions must be fulfilled. These include (a) effective organisation of protein molecules on a solid surface with sufficient stability against desorbing, (b) good electronic coupling between a protein molecule and external electrodes (i.e. electrical contacts to form molecular junctions), (c) control of molecular orientation and (d) conformation relevant to their biological structure and function under the experimental conditions required for measurements. These conditions have only been partially fulfilled in previous attempts. ${ }^{16-26}$ Conductance measurements on proteins by scanning probe techniques have nearly all involved a tunnelling barrier between tip and molecule, ${ }^{20-26}$ or the application of mechanical force to improve the electrical contact between tip and molecule. ${ }^{23}$ But recently, we have been able to measure STM conductance of protein 
molecules in air, ${ }^{27,28}$ contacted to two metallic electrodes via chemical anchoring groups. In the present study we show the first single-molecule electrochemical-STM conductance measurements of a heme protein, cytochrome $b_{562}$ (cyt $b_{562}$ ), of controlled orientation, and contacted directly via two thiol anchoring groups. By operating in solution under electrochemical control, the potential of the tip and substrate can be independently adjusted with respect to a pseudo-reference electrode in the solution. With fixed tip-substrate potential difference, the molecular conductance was found to depend strongly on the substrate potential, with the conductance of the equilibrium redox state being higher than any other reported molecule of comparable dimensions. The origin of this high conductance for the protein is discussed and related to its electron transfer behaviour.

\section{Results and discussion}

\subsection{Controlled protein orientation, conformation and electrode binding}

Cyt $b_{562}$ is an ET protein found in the periplasm of the bacteria Escherichia coli. The protein binds non-covalently the redoxactive iron protoporphyrin IX (heme) via two axial ligands. The central iron ion can switch between the $\mathrm{Fe}^{2+}$ and $\mathrm{Fe}^{3+}$ state, with the redox potential defined by heme-protein interactions. ${ }^{29}$ Heme binding is also critical to the stability of the protein's native conformation. In the absence of heme (apo-protein) the protein is partially folded and highly dynamic. ${ }^{26,29}$ Using rational protein engineering, pairs of cysteine residues were introduced that allow cyt $b_{562}$ to bind through metal-thiol interactions in defined orientations with respect to the electrode surface and an STM tip. ${ }^{27}$ Two sets of cysteine pairs were introduced to sample the two orientations of the approximately prolate spheroidal cyt $b_{562}$ : (a) the Cys21-Cys50 pair located at opposing ends of protein's long axis (denoted as SH-LA cyt $b_{562}$ ), leading to a tip-tosurface distance of $\sim 5.2 \mathrm{~nm}$ (Fig. 1(a), left) and (b) the Cys5Cys104 pair binding across the short axis of the protein (SH-SA cyt $b_{562}$ ) leading to a tip-to-surface distance of $\sim 2.4 \mathrm{~nm}$ (Fig. 1(a), right). Cyt $b_{562}$ with only the Cys50 mutation (D50C) was constructed as a control to confirm the effects of direct linkage of the cysteine pair protein variants to both tip and substrate. ${ }^{26}$ All the cysteine mutants bound heme in the same manner as the original wild-type protein, as verified by UVVisible spectroscopy and hemin titration experiments. ${ }^{27}$

Protein together with the reducing agent 1,4-dithiothreitol (DTT), which prevents formation of disulphide bonds, was absorbed on a gold surface to form a mixed self-assembled monolayer. ${ }^{26}$ All the measurements were performed in a pseudobiological buffer (sodium phosphate, $\mathrm{pH}$ 6.2) at room temperature $\left(23 \pm 2{ }^{\circ} \mathrm{C}\right)$. These combined experimental conditions create a well-defined environment compatible with the protein retaining its native structure and ET function for single-molecule measurements. ${ }^{26}$

Cyt $b_{562}$ deposition on $\mathrm{Au}(111)$ substrates produced species with defined molecular orientations in line with positioning of the cysteine residues and with dimensions consistent with retention of their native structure. STM images (Fig. 1(b)) obtained at different surface locations show well-dispersed protein (a)
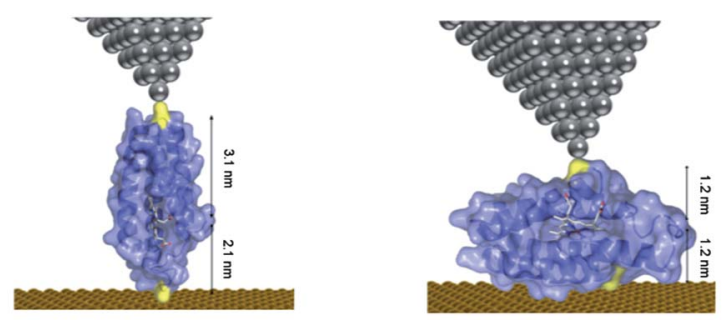

(b)
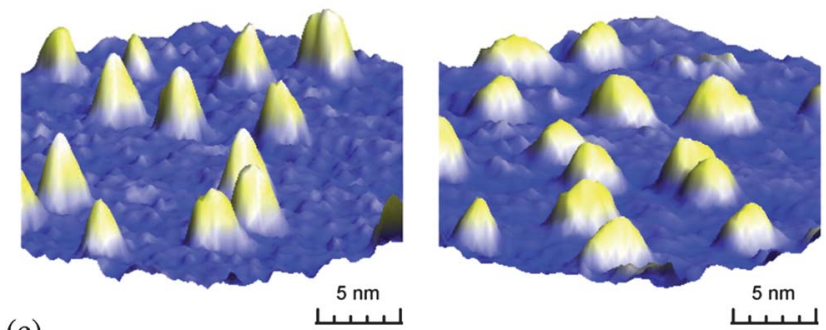

(c)
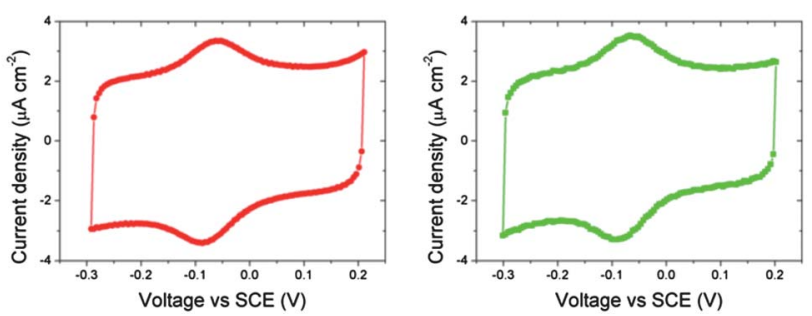

Fig. 1 Self-assembly, STM imaging and long-range protein electron transfer. (a) Schematic illustrations of self-assembly of SH-LA (left) and SH-SA (right) cyt $b_{562}$ molecules on a $\mathrm{Au}(111)$ surface in the two distinct orientations; (b) 3D view of STM images of cyt $b_{562}$ SH-LA (left) and SH$\mathrm{SA}$ (right) recorded in sodium phosphate buffer $\left(10 \mathrm{mM}, \mathrm{pH}\right.$ 6.2). $I_{\mathrm{t}}=35$ $\mathrm{pA}, V_{\mathrm{b}}=-0.4 \mathrm{~V}, E_{\mathrm{w}}=-0.10 \mathrm{~V}(v s$. SCE), $z$-range $=0.5 \mathrm{~nm}$; (c) cyclic voltamograms of cyt $b_{562}$ SH-LA (left) and SH-SA (right) recorded in the same conditions as for STM imaging with a scan rate of $0.5 \mathrm{~V} \mathrm{~s}^{-1}$.

molecules. The observed lateral dimensions of the imaged single molecules of either cyt $b_{562}$ SH-LA (circular, with diameter $3.2 \pm$ $0.5 \mathrm{~nm}$, Fig. 1(b), left) or SH-SA (laterally elongated, with a long axis of $4.5 \pm 0.7 \mathrm{~nm}$, Fig. 1(b), right) are in good agreement with crystallographic and NMR data for the holo-protein. ${ }^{29,30}$ (ESI, Fig. S1 $\uparrow$ presents a more detailed analysis of molecular dimensions.) In contrast, the apo-protein did not retain its native structure on adsorption onto the gold surface (ESI, Fig. S2 $†$ ), in keeping with the protein being only partially folded and highly dynamic in solution. ${ }^{26}$ Cyclic voltammograms in Fig. 1(c) demonstrate reversible ET between the protein and the electrode surface, which is essential for precise control of the protein redox state by electrochemical potentials applied to a working electrode or a STM substrate. No Faradaic response was observed in any of the cyclic voltammetry control measurements performed with apo-SH-SA, apo-SH-LA or holo-wild-type cyt $b_{562}$ (ESI, Fig. S3 to $S 6 \dagger)$. The measured equilibrium potential $E^{0^{\prime}}$ of $-68 \pm 2 \mathrm{mV}$ $v s$. SCE for both cyt $b_{562}$ SH-LA and cyt $b_{562}$ SH-SA variants is very close to those reported for the wild-type cyt $b_{562}$ adsorbed onto hydrophobically modified silver and on the $\mathrm{Au}(111)$ surface modified with a peptide linker monolayer ${ }^{31}$ The electrochemical measurements together with the measured protein dimensions confirm that the protein retains its functional conformation 
when adsorbed onto the Au(111) surface via an introduced cysteine residue.

\subsection{Electrochemical gating of single cyt $b_{562}$ protein molecules}

The apparent height of individual molecules, obtained from direct STM imaging, was found to depend on redox overpotential. Fig. 2(a) shows a series of STM images of cyt $b_{562}$ SH-SA molecules recorded with the bias voltage $V_{\mathrm{b}}=V_{\mathrm{t}}-V_{\mathrm{s}}$ between the tip (voltage $V_{\mathrm{t}}$ ) and the substrate (voltage $V_{\mathrm{s}}$ ) kept constant while adjusting the substrate potential relative to the reference electrode to control the redox state of the proteins. Apparent height versus overpotential $\eta=V_{\mathrm{s}}-E^{0^{\prime}}$ in Fig. 2(b) clearly shows, as the redox state of the protein molecules is shifted relative to the Fermi energy of the metal substrate, a peak in the apparent height for both the SH-LA and SH-SA variants. The maximum in apparent height is, as observed in related experiments, ${ }^{16,17,26}$ close to the protein equilibrium redox potential $\eta=0$. No change in lateral dimension was seen. STM-derived heights in proteins are known to be lower than the true height due to a relatively large separation between tip and surface during STM imaging at low set-point current, with only a slight lifting of the tip as it passes over the less conducting molecule. ${ }^{21}$ Variation in protein conductance rather than any significant protein conformational change is the likely dominant factor affecting the apparent height ${ }^{9,21}$ as also confirmed by tunnelling current-distance results discussed below. These results can be described quantitatively with a protein conductance model of 'two-step' ET between tip and substrate through the redox level of the protein. ${ }^{15-17,26}$ In Fig. 3 we schematically illustrate the energy alignment of redox level, substrate and tip in the two-step redox processes. The current $I_{\mathrm{t}}$ can be expressed:

$$
I_{\mathrm{t}}=2 e n_{\mathrm{el}} \frac{k_{\mathrm{sm}} k_{\mathrm{mt}}}{k_{\mathrm{sm}}+k_{\mathrm{mt}}}
$$

where $e$ is the electronic charge, $k_{\mathrm{sm}}$ and $k_{\mathrm{mt}}$ are the rate constants for ET between redox molecule and substrate (i.e. working electrode) and between STM tip and redox molecule, respectively, $n_{\mathrm{el}}$ is the number of electrons coherently transferred whilst the redox level relaxes through the energy window $e V_{\mathrm{b}}$ between the Fermi levels of the tip and substrate. ${ }^{32}$ The term $n_{\mathrm{el}}$ describes the fact that, in contrast to the normally encountered case of redox ET between a metal electrode and a molecular electronic level, in the STM junction there is a strong coupling to two electrodes (tip and substrate). A large number of electrons is thus transferred via the molecular level between the electrodes before the reduced molecular level has time to reach its relaxed state, which may be below the Fermi level of the other electrode. (We picture just the initially oxidised state for ease of description, but this applies similarly for an initially reduced state.) This transfer can occur because within the energy window $e V_{\mathrm{b}}$ there is a quasi-continuum of available empty states in one metal which can accept an electron, and full states in the other metal which can provide an electron. The rate at which the molecule can be oxidised/reduced by an electrode (which is limited by nuclear reorganisation processes) therefore does not limit the STM current for the strong coupling case, although it does influence the overall current. Using standard expression for rate constants, ${ }^{16}$ eqn (1) takes the following form at small overpotentials and bias voltages $\left(|e \xi \eta|,\left|e \gamma V_{\mathrm{b}}\right| \leq \lambda\right)$ :

$$
\begin{aligned}
I_{\mathrm{t}}= & I_{0} n_{\mathrm{el}}\left\{\exp \left[\frac{\left(\lambda+e \xi \eta+e \gamma\left|V_{\mathrm{b}}\right|\right)^{2}}{4 \lambda k_{\mathrm{B}} T}\right]\right. \\
& \left.+\exp \left[\frac{\left(\lambda-e \xi \eta-e \gamma\left|V_{\mathrm{b}}\right|+e\left|V_{\mathrm{b}}\right|\right)^{2}}{4 \lambda k_{\mathrm{B}} T}\right]\right\}^{-1}
\end{aligned}
$$
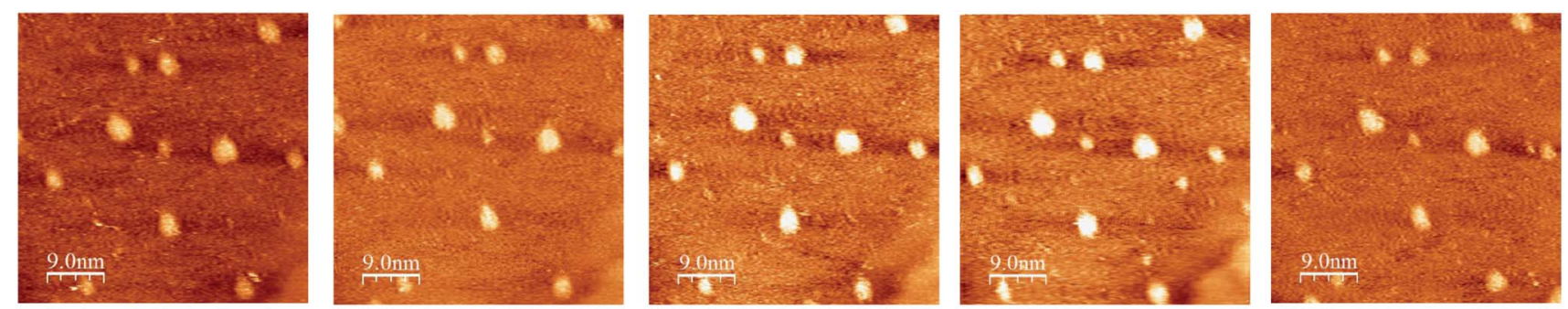

(a)
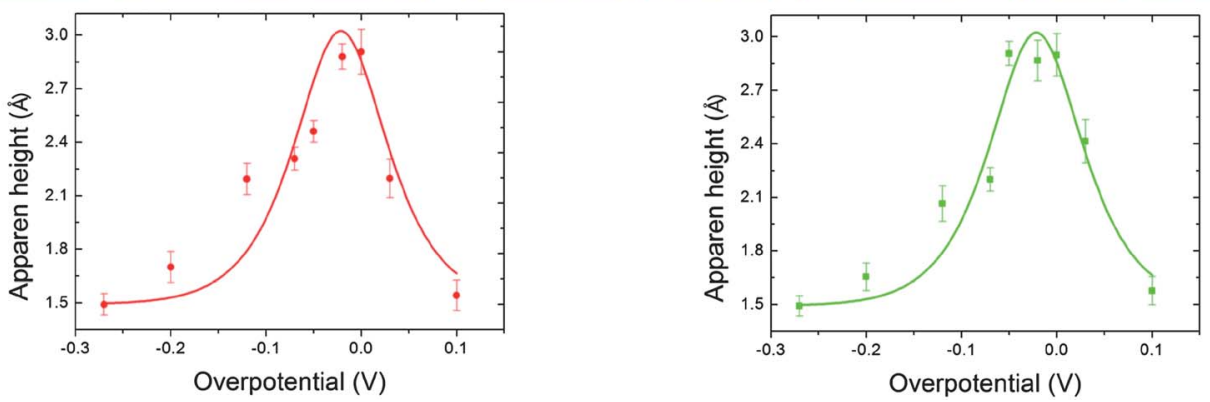

Fig. 2 In situ single-molecule mapping of redox state-dependent conductance. (a) Series of STM images for SH-SA molecules obtained at different overpotentials $\left(I_{\mathrm{t}}=35 \mathrm{pA}, V_{\mathrm{b}}=-0.4 \mathrm{~V}, \eta=E_{\mathrm{s}}-E^{0^{\prime}}=-210,-100,-10,+80,+130 \mathrm{mV}\right.$ from left to right, scan area $45 \times 45 \mathrm{~nm}, z$-range $\left.0-1.5 \mathrm{~nm}\right)$. (b) Dependence of apparent contrasts of SH-LA (left) and SH-SA (right) on the overpotentials applied to the working substrate electrode. The solid lines indicate theoretical fitting according to eqn (2) described in the text. Fitted parameters $\xi=1, \gamma=0.5, \lambda=0.10 \pm 0.03 \mathrm{eV}$ and $\lambda=0.15 \pm 0.03 \mathrm{eV}$ for $\mathrm{SH}-$ SA and SH-LA respectively. The measured vertical height of the protein in STM scans does not represent the true height of the protein ${ }^{21}$ due to the low conductance of biological molecules compared with the substrate, and the properties of the surrounding medium. ${ }^{22-25}$ 

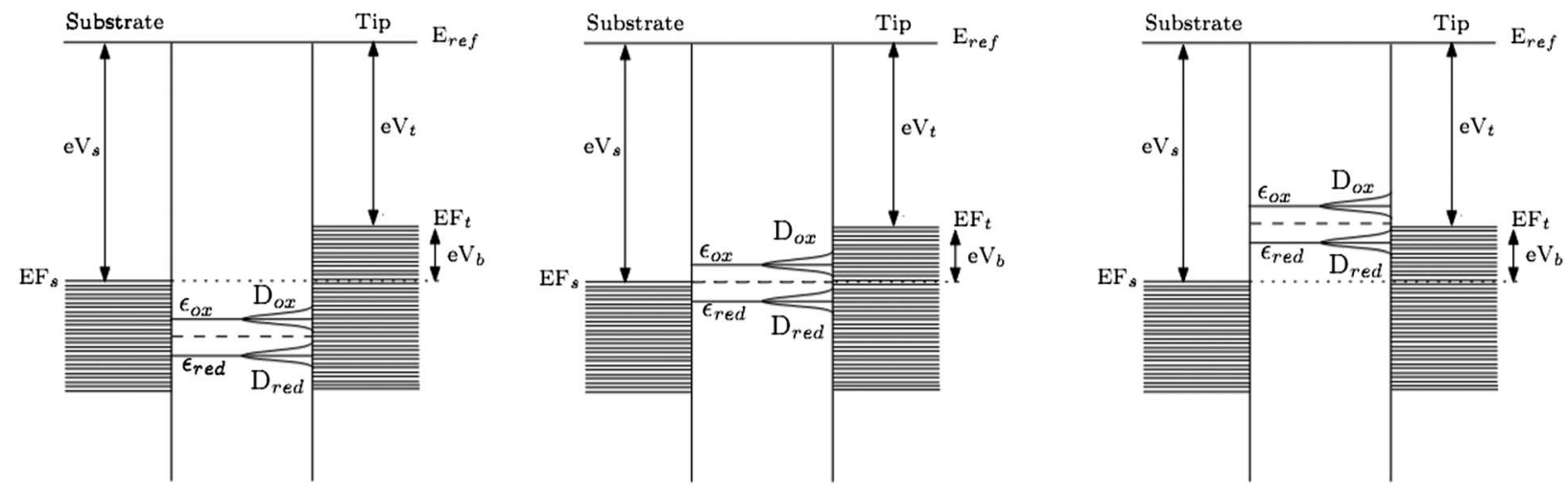

Fig. 3 Energy diagrams describing the sequential two step electron transport mechanism. The tip and the substrate Fermi levels $\left(\mathrm{EF}_{\mathrm{t}}\right.$ and $\left.\mathrm{EF}_{\mathrm{s}}\right)$ are separated by energy equal to $e V_{\mathrm{b}}$ which is kept constant through the experiment. The two Fermi energies $\mathrm{EF}_{\mathrm{t}}$ and $\mathrm{EF}_{\mathrm{s}}$ can be shifted in parallel with respect to the reference Fermi level tuning the substrate electrode potential. The dotted line, centred at the cyt $b_{562}$ equilibrium potential, indicates the protein redox state. Depending on the applied bias, electrons tunnel from one electrode to the other via the redox molecular level. If the protein equilibrium energy is aligned to the substrate Fermi level ( $\eta=0$, middle diagram), electrons transfer first from the tip to a molecular vacant state and, whilst the level relaxes vibrationally below the tip Fermi energy, to the substrate. The probability of resonant tunnelling from one electrode to the other decreases when the molecular equilibrium level is brought below (left diagram) or above (right diagram) the metallic Fermi level by applying a negative and a positive overpotential to the system, respectively. The model shows that, by changing the working electrode potential, the molecular redox states can be modulated in and out of resonance, resulting in increase and decrease of molecular conductance.

where $\lambda$ is the total nuclear (protein and solvent) reorganisation free energy, $\eta$ the substrate overpotential, $k_{\mathrm{B}}$ Boltzmann's constant and $T$ the temperature. The terms $\xi$ and $\gamma$ represent formally the fraction of the substrate potential and bias voltage drops at the site of the heme group redox centre. ${ }^{16,33} I_{0}$ is a constant, the most important part being the effective nuclear vibrational frequency of all the nuclear modes reorganised. The number $n_{\mathrm{el}}$ (which is proportional to $e V_{\mathrm{b}}$ ) is large when the electronic coupling between the molecule and the enclosing electrodes is strong and the adiabatic limit of interfacial ET prevails, as in the present case.

Eqn (2) formally represents well the data from the STM contrast (solid lines in Fig. 2(b)). $\xi$ and $\gamma$ are not known exactly and are correlated in the confined space of the in situ STM tunnelling gap. ${ }^{16}$ As an illustration, if the redox center experiences all the substrate potential applied electrochemically $(\xi=1)$ and half of the bias voltage $(\gamma=0.5)$, the reorganisation free energy parameter $\lambda$ can be obtained from fitting, giving $\lambda=0.10$ $\pm 0.03 \mathrm{eV}$ and $\lambda=0.15 \pm 0.03 \mathrm{eV}$ for SH-SA and SH-LA, respectively. Even with correlations or non-ideal values of $\xi(<1)$, these fitted values of $\lambda$ represent upper limits on the reorganisation energy. Contributions to $\lambda$ from heme and other porphyrins have been determined experimentally and theoretically to be in the range $0.05-0.15 \mathrm{eV} .^{34-36}$ The low limiting value of $\lambda$ indicates that reorganisation of the heme occurs during ET but much of the reorganisation of the solvent and of the protein scaffold $(\lambda \approx$ $0.7-1.1 \mathrm{eV})^{37}$ is decoupled in the molecular scale solvent confinement of the STM tunnelling gap. We also note that transient spectroscopy measurements inevitably involve solvent reorganisation for surface-bound donors or acceptors. ${ }^{38}$ In contrast, in the STM measurement electrons are transferred between two free-electron reservoirs (metal substrate and tip, which essentially play the role of donor and acceptor of electrons during electron transfer) and their charge state is fixed by the applied bias. Thus reorganisation of the solvent surrounding the electrodes is not induced during electron transfer.

\subsection{High single molecule conductance of cyt $b_{562}$}

STM imaging gives an indirect measure of the electrochemical gating of the current through the protein via changes in image contrast. A more powerful approach is to quantify the current through the molecule versus applied bias or tip-surface distance. The latter, usually referred to as the $I_{\mathrm{t}}-z$ technique, is commonly used for small molecules and allows unambiguous identification of data where a junction is clearly formed between the molecule and both tip and surface. ${ }^{-14}$ Recently, the $I_{\mathrm{t}}-z$ measurements have been applied to extract valuable information on the ET process for the protein azurin ${ }^{22}$ bound to the surface through a single cysteine residue present in the protein, but without a defined interaction with the tip. In our present study we take advantage of the free thiol group to make $I_{\mathrm{t}}-z$ measurements which provides a defined covalent tip-molecule interaction and avoids a tunnelling barrier between tip and molecule, in the same manner that most small-molecule studies are performed. Furthermore, we ensure that only a single protein is measured at a time by imaging before and after the $I_{\mathrm{t}}-z$ scans.

Molecules were first identified by imaging a small scanned area of 10 or $20 \mathrm{~nm}^{2}$, (ESI, Fig. S7 $\dagger$ ) and the STM tip was then positioned above a protein molecule with small set point tunnelling current (e.g. $35 \mathrm{pA}$ at $-0.1 \mathrm{~V}$ ). After disengaging the STM feedback system, the change in $I_{\mathrm{t}}$ was recorded as a function of the tip displacement while the tip was repeatedly moved towards and away from the target molecule. As the tip is withdrawn, a plateau in the measured current may be observed in the $I_{\mathrm{t}}-z$ curve just prior to losing contact with the molecule, at which point the current decreases rapidly (Fig. 4(a), left and middle). No current plateaus were observed for D50C proteins having just one cysteine residue, bound to the Au surface (Fig. 4(a), right) or for $I_{\mathrm{t}}-z$ measurements away from proteins where the surface is only populated with 1,4-dithiothreitol molecules. Conductance histograms were constructed, without selection, from $I_{\mathrm{t}}-z$ data and are shown in Fig. 4(b). Approximately $20 \%$ of the $I_{\mathrm{t}}-z$ traces 

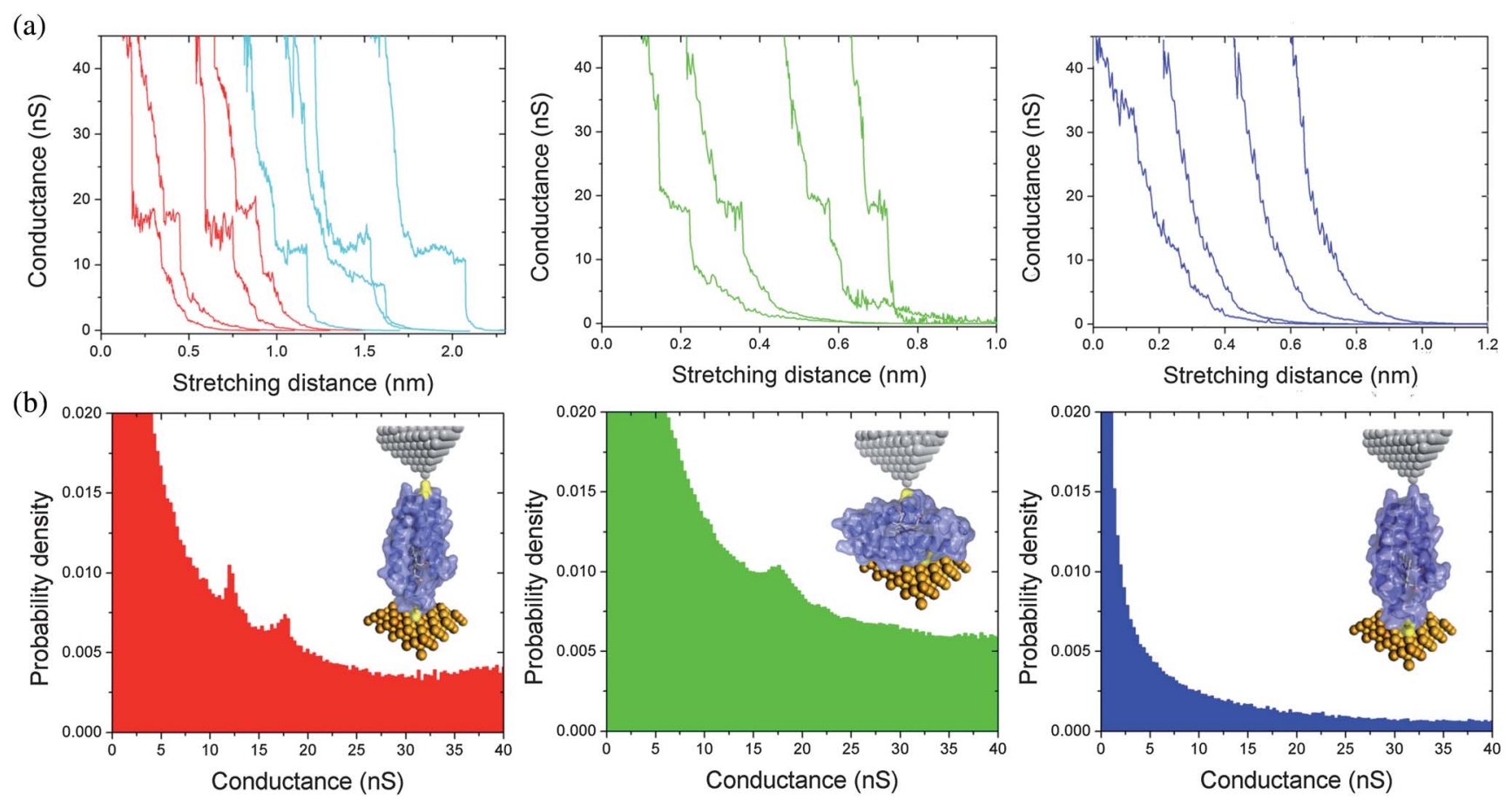

Fig. 4 Direct $I_{\mathrm{t}}-z$ measurements of protein conductance based on single-molecule junctions. (a) Representative $I_{\mathrm{t}}-z$ curves for SH-LA (left), SH-SA (middle), D50C molecules (right). (b) All-data histograms of molecular conductance. Each histogram was constructed from $I_{\mathrm{t}}-z$ curves on 12 SH-SA molecules ( 200 on each with a total of 2400 curves) and 11 SH-LA molecules ( 2200 curves) and 10 D50C molecules (2000 curves). No data selection was employed, but each $I_{\mathrm{t}}-z$ trace was weakly pre-smoothed as described in the ESI, $\uparrow$ where histograms based on un-smoothed traces are also presented (ESI, Fig. S9†). None of the D50C curves showed any evidence of plateaus. The measurements were performed in $10 \mathrm{mM}$ phosphate buffer (pH 6.2) at a fixed tip-substrate bias voltage of $-0.1 \mathrm{~V}$, and at the equilibrium redox potential of the protein molecules (i.e. $\eta=0 \mathrm{mV}$ ).

were non-exponential and exhibited plateaus with length ranging between 0.05 and $0.3 \mathrm{~nm}$. These current plateaus are assigned to the cleavage of individual protein-tip junctions. Around $70 \%$ of traces failed to form a molecular junction and showed a monotonic exponential decay, which represents direct electron tunnelling from the STM tip. The remaining $10 \%$ of traces were too noisy to classify. The step lengths observed in the $I_{\mathrm{t}}-z$ traces are small $(0.1-0.2 \mathrm{~nm})$ compared with the molecular length, as is expected for these protein molecules since they bind to the surface in defined orientations, rather than lying more nearly flat on the surface as for measurements on conventional larger organic single molecules. In the latter case, there can be a larger range of distance over which the tip binds to one end of the molecule (as the tip is withdrawn from the surface and the angle between molecule and surface is altered) and so lead to a long plateau. One consequence of small step length is that peaks in the histograms are less pronounced.

The conductance histogram for cyt $b_{562}$ SH-LA shows a main peak at $12.0 \mathrm{nS}$ accompanied by a smaller peak at $17.8 \mathrm{nS}$, while only a single distinct peak at $17.8 \mathrm{nS}$ is observed for cyt $b_{562} \mathrm{SH}$ SA. The observation of two conductance values for cyt $b_{562} \mathrm{SH}-$ LA is unlikely to correspond to one and two protein molecules, since the higher conductance value is not simply double that of the lower and imaging of well-separated protein molecules was achieved. Similar features have been observed in several small organic molecular systems ${ }^{39-41}$ where it is known that the contact configuration is a factor in determining conductance. ${ }^{14}$ Another possible interpretation ${ }^{27}$ is that the two conductance values correspond to the two possible orientations of the SH-LA molecule (Fig. 1(a), left) on the surface, since the iron redox centre of the heme is asymmetrically positioned along the protein's long axis. In contrast, the two possible orientations for cyt $b_{562}$ SH-SA have similar distances ${ }^{30}$ between the heme centre and either thiol group, see Fig. 1(a). Further experimental and theoretical work is required to test these interpretations.

The $I_{\mathrm{t}}-z$ measurements were also performed at various substrate potentials to observe redox-state dependent conductance. Fig. 5 shows the conductance histograms for cyt $b_{562} \mathrm{SH}-$ SA in (a) the fully oxidised form, (b) at the equilibrium redox potential, and (c) in the fully reduced form. It is clear that the protein in its equilibrium state is significantly more conducting than in its fully oxidised and fully reduced form. This strong overpotential dependence of the conductance in $I_{\mathrm{t}}-z$ measurements confirms that the apparent height dependence on overpotential cannot purely be attributed to conformational changes. A comparison of our results with those for selected molecules of similar length is summarised in Table 1. Combined with the results for SH-LA, this suggests that the placement of the iron moiety in the context of the protein plays a crucial role in ET. It is immediately noticeable that cyt $b_{562}$ is highly conductive, even in its oxidised and reduced states. Its conductance is large compared to that for a dsDNA fragment or a conjugated polymer wire of similar length. In particular, the conductance of cyt $b_{562}$ in its equilibrium state approaches 10 times higher than that of a single zinc porphyrin monomer with two thiol contact groups (ca. $2.2 \mathrm{nS}) .{ }^{43}$ The conductance values of SH-LA and 

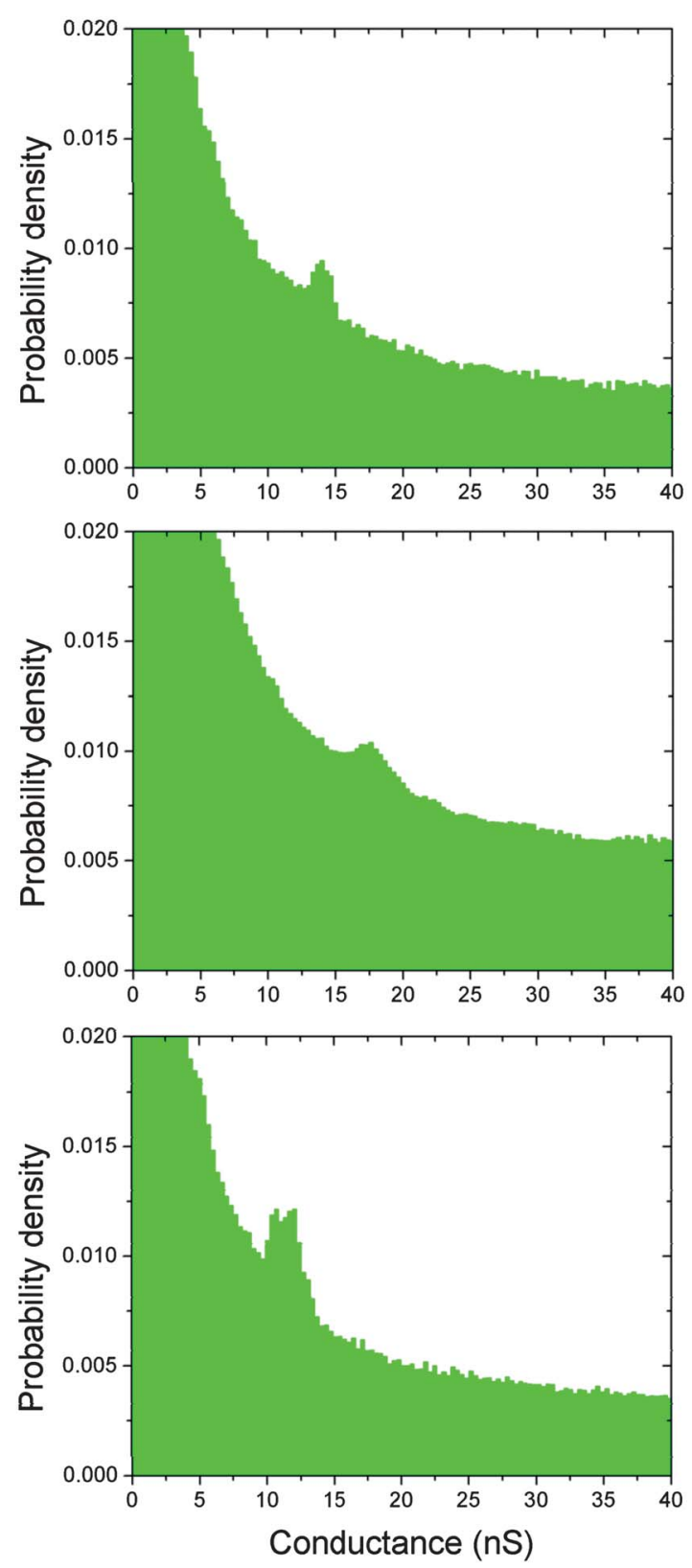

Fig. 5 Single-molecule conductance modulation of cyt $b_{562}$ SH-SA by its redox state. The redox state of the protein was controlled by the working substrate potentials applied: (top) the oxidised form $(\eta=+300 \mathrm{mV})$, (middle) the equilibrium state $(\eta=0 \mathrm{mV})$, and (bottom) the reduced form $(\eta=-300 \mathrm{mV})$. The measurements were performed in $10 \mathrm{mM}$ phosphate buffer (pH 6.2) at a fixed bias voltage of $-0.1 \mathrm{~V}$.

SH-SA cyt $b_{562}$ are around one order of magnitude greater than that measured for azurin, another ET protein, ${ }^{18,22,50}$ having a single cysteine linker to the gold electrode. This is consistent with the observation that adding a second thiol contact between a measured molecule and the STM tip increases the measured conductance by an order of magnitude or more. ${ }^{6,51}$ The somewhat lower cyt $b_{562}$ conductance values measured in air $^{27}$ of 1.5 and $2.7 \mathrm{nS}$ for the SH-LA and SH-SA molecules respectively might arise from a number of factors. For example, solvent has been reported to change the $\mathrm{Au}$ effective work function ${ }^{52}$ and increase single molecule conductance. The protein charge distribution and reorganisation energy, whose influence on the measured conductance is difficult to estimate, may also be affected. Strong solvent effects have been observed in some molecules. ${ }^{42}$

Cyclic voltammetry measurements on azurin give ET rates ${ }^{53,54}$ around $10^{2}$ to $10^{3} \mathrm{~s}^{-1}$, which is much lower than might be expected on the basis of the STM measurements. However, our EC-STM measurements involve strong coupling to two electrodes, whereas the measured transfer rate for voltammetry is limited by the lowest value of the ET rate between molecule and electrode, between molecule and solution (necessarily involving solvent reorganisation) and the inverse charging time constant of the double layer. Meaningful comparisons require ultra-fast voltammetry. ${ }^{55}$

The nature of this high conductance for cyt $b_{562}$ can be appreciated by the following considerations. Thiol linkers vastly increase conductivity of metal-molecule-metal junctions, and thiolated molecules are now ubiquitous in non-protein singlemolecule electronics. The mechanism of the improved conductivity is the stronger quantum mechanical coupling to both tip and substrate of the chemisorbed molecular levels, ${ }^{56,57}$ resulting in a significant broadening $\Delta E$ in their energy. Our results suggest that this is the case for the heme level of the proteins, although this requires verification by detailed electronic structure calculations since $\Delta E$ depends sensitively on the molecular length, details of the contact and nature of the molecule. From the Heisenberg uncertainty relation $\Delta E \Delta t \sim \hbar$ the lifetime of this state $\Delta t$, which we denote as $t_{\mathrm{el}}$, governs the intrinsic ET rate $1 / t_{\mathrm{el}}$, as outlined by Datta. ${ }^{58} \mathrm{~A}$ broadening of around $1 \mathrm{meV}$, as found for example in DFT calculations ${ }^{59}$ of the porphyrin monomer of Table 1, corresponds to a rate of $10^{12} \mathrm{~s}^{-1}$. A crude estimate based on the $\beta$ factor of the $I_{\mathrm{t}}-z$ curves $^{60}$ is also consistent with a broadening of 1 and $10 \mathrm{meV}$, for the LA and SA molecules. There are uncertainties in such estimates, and this value might easily be exceeded. ${ }^{9}$

In terms of the two-step model above, when the relevant redox state lies between the Fermi energies of tip and substrate, electrons are transferred at this rate between molecule and tip/ substrate. The number of electrons that are transferred in a single vibrational relaxation passage of the molecular redox state through the energy window between the substrate and tip Fermi levels depends on the nuclear environment. This is characterised by a vibrational relaxation time $t_{\text {rel }}$ that includes all the classical vibrational modes of the solvent that are dominated by low frequency relaxation, ${ }^{16}$ giving altogether $t_{\text {rel }}$ $\sim 10^{-12} \mathrm{~s}$. This value includes a spectrum of values and could be larger if slow protein conformational modes and solvent confinement in the tunnelling gap are also included. If the redox level relaxes slowly enough (large $t_{\text {rel }}$ ) and the broadening is strong enough, then the ratio $t_{\text {rel }} / t_{\mathrm{el}}$ can become large and many electrons are transferred whilst the level relaxes. The diabatic limit of relatively weak interaction corresponds to $\Delta E \sim 10^{-4}$ to $10^{-3} \mathrm{eV}$ or $t_{\mathrm{el}} \sim(10-1) \times 10^{-12} \mathrm{~s}$. Larger values take us into the adiabatic range: ${ }^{32}$ in the adiabatic limit we could take $\Delta E \sim$ $10^{-2}$ to $10^{-1} \mathrm{eV}$ giving $t_{\mathrm{el}} \sim(10-1) \times 10^{-14} \mathrm{~s}$. Even for moderately adiabatic transitions the ratio $t_{\mathrm{rel}} / t_{\mathrm{el}}$ is thus likely to assume significant values (orders of magnitude), allowing for the coherent transfer of a large number of electrons in a single in situ STM event. 
Table 1 Comparison of single-molecular conductance of cyt $b_{562}$ with selected systems ${ }^{a}$

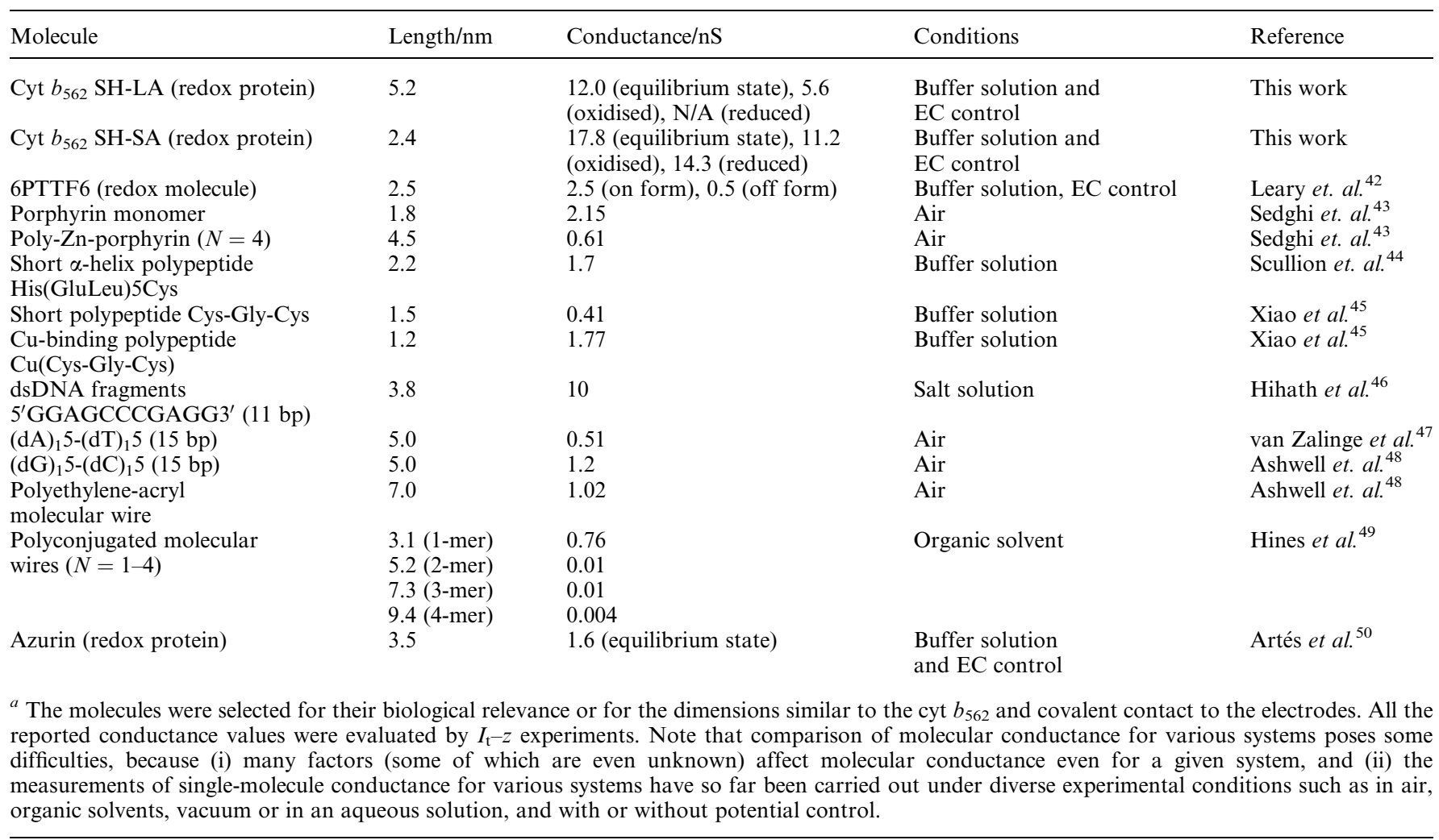

\section{Conclusions}

In summary, we present the first single-molecule conduction measurement of a protein chemically coupled, with controlled protein orientation, to two enclosing electrodes under pseudophysiological conditions with electrochemical control. Measured electrochemical equilibrium potentials $(-68 \pm 2 \mathrm{mV} v s$. SCE) are consistent with the native cyt $b_{562}$ conformation. Electrochemical gating of the molecular conductance was demonstrated both indirectly from apparent height contrast and directly from the $I_{\mathrm{t}^{-}}$ $z$ curves. The latter measurements showed that cyt $b_{562}$ has notably higher conductance for two controlled molecular orientations than those of comparable synthetic molecules of similar dimensions. The maximum conductance at the cyt $b_{562}$ electrochemical potential is consistent with its suggested role as an electron shuttle in the inter-membrane region, in which it can be oxidised or reduced according to the electrochemical potential of its interacting molecule. Two possible origins of the high conductance are (i) the solvent contribution to the reorganisation free energy is notably reduced, the main contribution being attributed to the heme, (ii) the strong electronic coupling due to the double metal-sulfur bond gives strongly adiabatic ET at both contacts and a multi-electron transfer via the heme redox level. The observation of ET in which solvent reorganisation is significantly reduced calls for a new systematic study of reorganisation energy in redox proteins, complementing traditional photoexcitation experiments of a surface-bound donor group. The methods developed here hold promise for effective new ways for single-molecule protein ET studies broadly, with prospects for incorporation of single ET protein molecules in its native conformation in an external electronic circuit for future nanoscale bioelectronic devices.

\section{Acknowledgements}

This work was supported in the UK by the BBSRC (Grant BB/ E001084 to D.D.J.) and EPSRC (Grant EP/D076072/1 to J.E.M. and M.E.) with ongoing support from EPSRC (Grant EP/ J015318/1 to M.E., J.E.M. and D.D.J.); in Denmark by the Danish Research Council for Technology and Production Sciences (Contract no. 274-07-0272 to Q.C. and J.U.) with ongoing support by the Lundbeck Foundation (Grant no. R49A5331, to Q.C. and J.U.). E.A.D.P. acknowledges the Cardiff University Richard Whipp Interdisciplinary Research Scholarship for Ph.D. study and the Charles Coles Travelling Scholarship for visiting Denmark. We thank Dr Jingdong Zhang for assistance in the STM experiments. The authors are grateful to several leading scientists in the related fields for reading the manuscript, stimulating discussions, and invaluable suggestions.

\section{References}

1 A. Messerschmidt, Handbook of Metalloproteins, Wiley, Chichester, 2001.

2 J. Berg, J. Tymoczko and L. Stryer, Biochemistry: International Edition, W. H. Freeman and Company, 2011.

3 H. B. Gray and J. R. Winkler, Annu. Rev. Biochem., 1996, 65, 537561.

4 H. B. Gray and J. R. Winkler, Q. Rev. Biophys., 2003, 36, 341-372.

5 C. C. Page, C. C. Moser, X. Chen and P. L. Dutton, Nature, 1999, 402, 47-52. 
6 X. D. Cui, A. Primak, X. Zarate, J. Tomfohr, O. F. Sankey, A. L. Moore, T. A. Moore, D. Gust, G. Harris and S. M. Lindsay, Science, 2001, 294, 571-574.

7 B. Xu and N. J. Tao, Science, 2003, 301, 1221-1223.

8 W. Haiss, H. van Zalinge, S. J. Higgins, D. Bethell, H. Höbenreich, D. J. Schiffrin and R. J. Nichols, J. Am. Chem. Soc., 2003, 125, 15294-15295.

9 N. J. Tao, Phys. Rev. Lett., 1996, 76, 4066-4069.

10 N. J. Tao, Nat. Nanotechnol., 2006, 1, 173-181.

11 W. Haiss, C. Wang, I. Grace, A. S. Batsanov, D. J. Schiffrin, S. J. Higgins, M. R. Bryce, C. J. Lambert and R. J. Nichols, Nat. Mater., 2006, 5, 995-1002.

12 F. Chen, J. Hihath, Z. Huang, X. Li and N. J. Tao, Annu. Rev. Phys. Chem., 2007, 58, 535-564.

13 S. Lindsay and M. Ratner, Adv. Mater., 2007, 19, 23-31.

14 R. J. Nichols, W. Haiss, S. J. Higgins, E. Leary, S. Martin and D. Bethell, Phys. Chem. Chem. Phys., 2010, 12, 2801-2815.

15 T. Albrecht, K. Moth-Poulsen, J. r. B. Christensen, J. Hjelm, T. Bjørnholm and J. Ulstrup, J. Am. Chem. Soc., 2006, 128, 6574-6575.

16 J. Zhang, A. M. Kuznetsov, I. G. Medvedev, Q. Chi, T. Albrecht, P. S. Jensen and J. Ulstrup, Chem. Rev., 2008, 108, 2737-2791.

17 Q. Chi, O. Farver and J. Ulstrup, Proc. Natl. Acad. Sci. U. S. A., 2005, 102, 16203-16208.

18 A. Alessandrini, S. Corni and P. Facci, Phys. Chem. Chem. Phys., 2006, 8, 4383-4397.

19 J. J. Davis, B. Peters and W. Xi, J. Phys.: Condens. Matter, 2008, 20, 374123.

20 L. Andolfi and A. Bizzarri, Appl. Phys. Lett., 2006, 89, 183125.

21 A. V. Patil and J. J. Davis, Coord. Chem. Rev., 2011, 255, 1970-1980.

22 J. M. Artés, I. Díez-Pérez, F. Sanz and P. Gorostiza, ACS Nano, 2011, 2060-2066.

23 J. J. Davis and H. A. O. Hill, Chem. Commun., 2002, 393-401.

24 Q. Chi, J. Zhang, J. Nielsen, E. Friis, I. Chorkendorff, G. Canters, J. Andersen and J. Ulstrup, J. Am. Chem. Soc., 2000, 122, 4047-4055.

25 D. Alliata, L. Andolfi and S. Cannistraro, Ultramicroscopy, 2004, 101, 231-240.

26 E. A. Della Pia, Q. Chi, D. D. Jones, J. E. Macdonald, J. Ulstrup and M. Elliott, Nano Lett., 2011, 11, 176-182.

27 E. A. Della Pia, M. Elliott, D. D. Jones and J. E. Macdonald, $A C S$ Nano, 2012, 6, 355-361.

28 E. A. Della Pia, J. E. Macdonald, M. Elliott and D. D. Jones, Small, 2012, 8, 2341-2344.

29 K. Hamada, P. H. Bethge and F. S. Mathews, J. Mol. Biol., 1995, 247, 947.

30 F. Arnesano, L. Banci, I. Bertini, J. Faraone-Mennella, A. Rosato, P. D. Barker and A. R. Fersht, Biochemistry, 1999, 38, 8657-8670.

31 P. D. Barker, J. L. Butler, P. de Oliveira, H. A. O. Hill and N. I. Hunt, Inorg. Chim. Acta, 1996, 252, 71-77.

32 J. Zhang, Q. Chi, A. M. Kuznetsov, A. G. Hansen, H. Wackerbarth, H. E. M. Christensen, J. E. T. Andersen and J. Ulstrup, J. Phys. Chem. B, 2002, 106, 1131-1152.

33 I. V. Pobelov, Z. Li and T. Wandlowski, J. Am. Chem. Soc., 2008, 130, $16045-16054$.

34 X. Amashukeli, N. E. Gruhn, D. L. Lichtenberger, J. R. Winkler and H. B. Gray, J. Am. Chem. Soc., 2004, 126, 15566-15571.

35 E. Sigfridsson and M. Olsson, J. Phys. Chem. B, 2001, 105, 55465552.
36 V. Tipmanee, H. Oberhofer, M. Park, K. S. Kim and J. Blumberger, J. Am. Chem. Soc., 2010, 132, 17032-17040.

37 C. Page, Curr. Opin. Chem. Biol., 2003, 7, 551-556.

38 J. Blumberger and M. L. Klein, J. Am. Chem. Soc., 2006, 128, 13854 13867.

39 J. He, O. Sankey, M. Lee, N. Tao, X. Li and S. Lindsay, Faraday Discuss., 2006, 131, 145.

40 R. Huber, M. T. González, S. Wu, M. Langer, S. Grunder, V. Horhoiu, M. Mayor, M. R. Bryce, C. Wang, R. Jitchati, C. Schönenberger and M. Calame, J. Am. Chem. Soc., 2008, 130, 1080-1084.

41 N. Bennett, G. Xu, L. J. Esdaile, H. L. Anderson, J. E. Macdonald and M. Elliott, Small, 2010, 5, 2604-2611.

42 E. Leary, S. J. Higgins, H. van Zalinge, W. Haiss, R. J. Nichols, S. Nygaard, J. O. Jeppesen and J. Ulstrup, J. Am. Chem. Soc., 2008, 130, 12204-12205.

43 G. Sedghi, K. Sawada, L. J. Esdaile, M. Hoffmann, H. L. Anderson, D. Bethell, W. Haiss, S. J. Higgins and R. J. Nichols, J. Am. Chem. Soc., 2008, 130, 8582-8583.

44 L. Scullion, T. Doneux, L. Bouffier, D. G. Fernig, S. J. Higgins, D. Bethell and R. J. Nichols, J. Phys. Chem. C, 2011, 115, 8361-8368. 45 X. Xiao, B. Xu and N. Tao, Angew. Chem., 2004, 43, 6148-6152.

46 J. Hihath, B. Xu, P. Zhang and N. Tao, Proc. Natl. Acad. Sci. U. S. A., 2005, 102, 16979-16983.

47 H. van Zalinge, D. J. Schiffrin, A. D. Bates, W. Haiss, J. Ulstrup and R. J. Nichols, ChemPhysChem, 2006, 7, 94-98.

48 G. J. Ashwell, B. Urasinska, C. Wang, M. R. Bryce, I. Grace and C. J. Lambert, Chem. Commun., 2006, 4706-4708.

49 T. Hines, I. Diez-Perez, J. Hihath, H. Liu, Z.-s. Wang, J. Zhao, G. Zhou, K. Müllen and N. Tao, J. Am. Chem. Soc., 2010, 132, $11658-11664$.

50 J. M. Artés, I. Díez-Pérez and P. Gorostiza, Nano Lett., 2012, 12, $2679-2684$.

51 A. Salomon, D. Cahen, S. Lindsay, J. Tomfohr, V. Engelkes and C. Frisbie, Adv. Mater., 2003, 15, 1881-1890.

52 V. Fatemi, M. Kamenetska, J. B. Neaton and L. Venkataraman, Nano Lett., 2011, 11, 1988-1992.

53 Q. Chi, J. Zhang, J. E. T. Andersen and J. Ulstrup, J. Phys. Chem. B, 2001, 105, 4669-4679.

54 L. J. C. Jeuken, J. P. McEvoy and F. A. Armstrong, J. Phys. Chem. B, 2002, 106, 2304-2313.

55 X.-S. Zhou, L. Liu, P. Fortgang, A.-S. Lefevre, A. Serra-Muns, N. Raouafi, C. Amatore, B.-W. Mao, E. Maisonhaute and B. Schöllhorn, J. Am. Chem. Soc., 2011, 133, 7509-7516.

56 F. Zahid, M. Paulsson and S. Datta, Advanced Semiconductors and Organic Nano-Techniques, Academic Press, 2003.

57 C. Li, I. Pobelov, T. Wandlowski, A. Bagrets, A. Arnold and F. Evers, J. Am. Chem. Soc., 2008, 130, 318-326.

58 S. Datta, Quantum Transport: Atom to Transistor, Cambridge University Press, 2005, p. 418

59 G. Sedghi, V. M. García-Suárez, L. J. Esdaile, H. L. Anderson, C. J. Lambert, S. Martín, D. Bethell, S. J. Higgins, M. Elliott, N. Bennett, J. E. Macdonald and R. J. Nichols, Nat. Nanotechnol., $2011,6,517-523$

60 E. H. Huisman, C. M. Guédon, B. J. van Wees and S. J. van Der Molen, Nano Lett., 2009, 9, 3909-3913. 